
\title{
25 Research Square \\ CSF3-Receptor Downregulation in Bone Marrow contributes to Ineffectiveness of GCSF in Advanced Liver Cirrhosis
}

Chhagan Bihari ( $\sim$ drcbsharma@gmail.com )

Institute of Liver and Biliary Sciences https://orcid.org/0000-0001-5801-9458

\section{Sukriti Baweja}

Institute of Liver and Biliary Sciences

Deepika Lal

Institute of Liver and Biliary Sciences

Seggere Murlikrishna Shasthry

Institute of Liver and Biliary Sciences Department of Hepatology

Preeti Negi

Institute of Liver and Biliary Sciences

Swati Thangariyal

Institute of Liver and Biliary Sciences

Dinesh Mani Tripathi

Institute of Liver and Biliary Sciences

Shiv Kumar Sarin

Institute of Liver and Biliary Sciences

\section{Research Article}

Keywords: Liver Cirrhosis, Child-Turcott-Pugh Score, Bone Marrow, Granulocyte-colony stimulating factor; CSF3R, CEACAM-1, Hematopoiestic Stem cells, Neutropenia, Leukopenia, SNPs

Posted Date: March 30th, 2021

DOl: https://doi.org/10.21203/rs.3.rs-347823/v1

License: (c) (i) This work is licensed under a Creative Commons Attribution 4.0 International License.

Read Full License 


\section{Abstract}

Introduction: Cirrhosis patients exhibit cytopenia and variable response to GCSF, with benefits in early cirrhosis. GCSF acts through the CSF3-receptor (CSF3R), and changes in CSF3R can affect the response. We investigated the possible mechanism for the lack of efficacy of GCSF in advanced cirrhosis

\section{Methods}

Cirrhotic patients $(n=127)$ and controls $(n=26)$ who underwent clinically indicated bone marrow (BM) examination were studied. BMs were assessed for RNA sequencing, QRT-PCR, and immunohistochemistry (IHC) for CSF3R and associated genes. Circulating GCSF, CSF3R, and carcinoembryonic antigen cell adhesion molecule-1 (CEACAM1) were measured. BM hematopoietic precursor cells and their alterations were examined by flow cytometry. The findings were validated, where the GCSF was administered as for liver regeneration $(n=22)$ and severe neutropenia $(n=15)$

\section{Results}

The mean age was $48.6 \pm 13.4$ years, $80.3 \%$ males. Gene set enrichment analysis showed lowered CSF3R in Child's $\mathrm{C}$ compared to A, confirmed by qRT-PCR and IHC. Circulatory CSF3R was also reduced in advanced cirrhosis. CSF3R decline was related to decrease hematopoietic stem cells (HSCs) and downregulation of CSF3R in remaining HSCs. CSF3R inhibitory protein CEACAM1 caused CSF3R downregulation. CEACAM-1, in turn, positively correlated with the increased lysosomal expression in HSCs. Peripheral blood-CSF3R was lesser in those cases who developed an infection Baseline CSF3R was also lower in G-CSF non-responders.

\section{Conclusions}

CSF3R is downregulated in patients with advanced cirrhosis, and it could be the underlying cause of the inadequate response to GCSF.

\section{Introduction}

Bone marrow (BM) and liver retain an evolutionary conserved developmental relationship. BM is the potential reservoir of hematopoietic stem cell (HSC), mesenchymal stem cell (MSC) populations, responds favorably to the peripheral tissue injury and various stress factors. It provides cells and specific molecules for tissue repair and regeneration. [1, 2] G-CSF stimulates neutrophil production and HSC mobilization both at basal and stress-induced granulopoiesis. It acts through the colony-stimulating factor receptor (G-CSFR), also known as CSF3R or CD114. CSF3R is primarily expressed in BM precursor cells, i.e., HSCs. Though it is a cell surface receptor, an extracellular domain shedding could be detected in the peripheral circulation. [3]

Clinical trials using G-CSF therapy have shown the survival benefit and efficacy to limit the complications in cirrhosis [4] and acute-on-chronic liver failure (ACLF) patients. [5] However, another study contradicted 
these findings. It showed more significant complications and mortality rates in the G-CSF plus stem cell infusion group. [6] Overall, the tangible benefits of G-CSF therapy remain variable and controversial.

Earlier, we have shown the quantitative and qualitative dysfunction of the BM stem cells in advanced cirrhosis. $[7,8]$ Other studies showed role of BM in hepatic regenerative response. $[9,10]$ A study from our group revealed that those with early cirrhosis and sufficient BM reserve could respond to GCSF. [11]

Another concern with the progressive stages of cirrhosis is severe cytopenia, including neutropenia. Sporadically, the neutropenia could be refractory to G-CSF. [12]

Considering these intriguing questions- (i) variable results to G-CSF therapy, (ii) effective response only in early cirrhosis, and (iii) at times cirrhosis related neutropenia; refractory to G-CSF led us to think whether these outcomes could be attributable to the variations \{quantitative and qualitative (single nucleotide polymorphism)\} in the CSF3R receptor. G-CSF's therapeutic impact is only justified if the receptor functioning is intact.

The present study aimed to assess the status of CSF3R in the BM precursor cells and its relationship with GCSF response.

\section{Patients And Methods}

Study Phases. The study conducted in 3 phases-

1. First, we analyzed 127 cirrhosis patients and 26 controls (details in the next section) for CSF3R and followed them for six months for naturally acquired infection.

2. Second, we validated these findings in two different clinical cohorts

- CSF3R receptor gene's variations for single nucleotide polymorphisms (SNPs) were analyzed in the third phase.

\section{Phase-1 study participants and methods}

Study group: The study included cirrhotic patients who underwent clinically indicated bone marrow (BM) examinations from Jan 2017 - June 2019 at the Institute of Liver and Biliary Sciences, New Delhi ( $n=127)$. BM examination was done as per the clinical indications \{(i.e., cytopenia, pyrexia of unknown origin, and dyserythropoiesis in refractory anemia in our previous study [13]\}. The clinical indications across the CTP A, B, and C categories were similar [Table S1]. Thus, the study population included all the CTP classes of patients, and hence the results could be generalizable beyond the study participants.

Control ( $n=26)$ : Non-liver disease subjects who underwent BM examination for other than liver disease or chronic ailment served as controls, as described. $[7,8]$ In these cases, the BM examination was done to investigate infiltrative or infective diseases. Those patients who did not have any systemic or BM pathology were considered as controls. 
Institutional ethics committee approval: The Institutional Ethics Committee of the Institute of Liver and Biliary Sciences, New Delhi, India, approved this study protocol (IEC/81/MA06). Informed consent was obtained from patients to use their leftover samples and clinical data after rendering the diagnosis. The leftover bone marrow aspirate and peripheral blood, plasma, and cells were isolated and stored with the clinical annotation in the National Liver Disease Biobank.

Bone Marrow Examination: BM aspirates and biopsies were examined for various hematopoietic cell morphologies.

Bone Marrow biopsies RNA sequencing: Details given in Suppl. Methods 1-2: Briefly, total RNA was isolated from five cases (control, Child-Turcott-Pugh Class A, and C). Total RNA was reverse transcribed, cDNAs library was prepared, and differential gene expression was analyzed in three groups- Control, Child-Turcott-Pugh Class A, and C.

Quantitative Reverse-Transcription Polymerase Chain Reaction: Details provided in suppl method-3. Briefly, total RNA was isolated, reversed transcribed into complementary DNA, and augmented by SYBR green. Transcripts were progressively expanded for gene-specific primers, listed in Table $\mathbf{S 2}$.

Bone Marrow Immunohistochemistry: BM biopsies were stained for colony-stimulating factor receptor 3 (CSF3R) (PAB476Hu01), hematopoietic cell kinase (HCK) (PAC522Hu01), splenic kinase (SYK) (PAE275Mu01), and carcinoembryonic antigen cellular adhesion molecule (CEACAM1) (MAC977Hu22) from Cloudclone, TX, USA. Cytoplasmic/surface positive cells were counted in consecutive 25 high-power fields $\left(20 x\right.$, area $\left.1.3 \mathrm{~mm}^{2}\right)$, and average numbers were calculated. Positive cells were assessed by Image $\mathrm{J}$ software.

Flow Cytometry assessment. Flow Cytometry assessment. Immunostaining was done using the whole bone marrow aspirate sample. After RBC lysis, cells were stained with CD45, CD34, CSF3R, and TLR3 (BD bioscience, CA, USA).

CFU-GM and BFU-E culture: Described in suppl. Method-4 Briefly, BM mononuclear cells (BMMNCs) were supplemented with erythropoietin for burst forming units-erythroid (BFU-E) and human granulocytemacrophage stimulating factor for colony-forming unit granulocyte-macrophage (CFU-GM).

Enzyme-Linked Immunosorbent Assay (ELISA): Enzyme-linked immunosorbent assay of human G-CSF (EEL-H0079), CSF3R (E-EL-H0799) from Elabscience, Hubei, CH; CEACAM-1 (CSB-EL005157HU), and Oncostatin M (CSB-E04696h), Cusabio, Hubei, CH was done on BM and peripheral blood (PB) plasma of cirrhosis and control cases.

\section{Phase-2 study groups}

BM and Peripheral blood plasma CSF3R at baseline were measured in two clinical cohorts. 
1. One cohort $(n=22)$ represents those cirrhotic patients who received G-CSF therapy in previous study [11] and where stored BM and peripheral blood plasma samples were available (retrospective). The response was considered if there is a reduction of CTP $>1$ and/or MELD $>2$ as described [14].

1. The second cohort was prospective, where G-CSF was given for severe neutropenia $(<1000 / \mathrm{cmm})$ in cirrhotic patients. We enrolled in these patients consecutively from June 2019 to June 2020 and assessed the response after 48 hours.

\section{Phase-3 study:}

Sanger sequencing for CSF3R was carried out on peripheral blood samples of phase 1 and 2 study subjects $(n=190)$ to discern the possible single nucleotide polymorphism in the CSF3R gene.

Statistical Analysis: Statistical analysis was carried out using Statistical Package for Social Sciences (SPSS, version 22.0) and MedCalc (version 20.0). Student's t-test/Mann-Whitney test was used to compare two groups based on the parametric data distribution (GSCF, CSF3R, CEACAM-1, and IHC) or non-parametric ( $\%$ cells). One-way ANOVA of variance for parametric data and Kruskal-Wallis test for non-parametric were applied for comparison amongst more than two groups. $\mathrm{P}<0.05$ was considered significant.

\section{Results}

\section{Primary attributes of study participants}

During Jan 2017 - June 2019, 268 BM examinations were done in the Institute of Liver and Biliary Sciences for various clinical indications. One hundred forty-five (145) were cirrhotic patients, and twentynine non-liver disease cases without pathology were considered controls. The rest of the cases $(n=94)$ belonged to other diseases and hence were excluded. BM of six cirrhosis patients and three controls were suboptimal, and 12 cirrhotic patients had associated neoplastic pathology and excluded from the study. [Study Participants Summarized in Fig S1].

Amongst the cirrhosis group, $28.3 \%$ patients belonged to CTP A ( $n=36), 42.6 \%$ to CTP B ( $n=54)$, and 29.1 $\%$ to CTP C $(n=37)$ classes. The most frequent etiology was alcohol-associated cirrhosis $(41.1 \%)$. These basic parameters amongst the CTP classes and controls succinctly summarized in Table-S3.

\section{Bone marrow transcriptomics showed Down-Regulation of CSF3R}

Bone marrow transcriptomics analysis showed that the immunological signatures are affected in cirrhotic patients. Especially, those related to innate immune responses become downregulated in advanced cirrhosis, i.e., CTP class C [Fig S2, S3]. Gene set enrichment analysis showed 294 downregulated genes [Fig.S4]. Specifically, 34 genes were downregulated across the group (Control>CTP A> CTP C) including CSF3R [Fig.1A]. Further, it was confirmed by qRT-PCR. Whereas, the associated genes like HCK and SYK were comparable amongst the CTP classes. [Fig. 1B]. Immunohistochemistry showed 
reduced CSF3R+ cells but not HCK and SYK positive cells/20X with cirrhosis progression. [Fig. 1C, D] These results indicate the reduced CSF3R expression in the BM precursor cells.

\section{CSF3R in Peripheral Blood}

Since bone marrow is routinely not done in cirrhotic patients, we decided to test the CSF3R levels in the peripheral blood plasma. We assessed the levels of both ligand (G-CSF) and CSF3R in the BM and peripheral blood plasma. The plasma levels of G-CSF were increased [Fig.2A], whereas the receptor levels were found to be lowered [Fig.2B], with the advancement of cirrhosis stages.

There was no difference in BM and peripheral blood of CSF3R $(p=0.439$ and 0.850$)$ amongst various cirrhosis etiologies. Also, BM and peripheral blood CSF3R ( $p=0.539$ and $p=0.131)$ were comparable among male and female patients.

During a follow-up of 6 months, 26 had naturally acquired infection, and of these, seven had pneumonia, urinary tract infection (06), spontaneous bacterial peritonitis (10), and other infections (03). Those patients who had developed infection had lesser BM-CSF3R $(p=0.056)$ and peripheral blood CSF3R $(p=0.022)$ levels as compared to the patients who did not. [Fig. 2C]. Similarly, patients with peripheral blood leukopenia (total leukocyte count $<4 \times 10^{\wedge} 6 / L, n=31$ ) also demonstrated lower levels of CSF3R in the BM plasma ( $p=0.001)$ and peripheral blood $(p=0.217)$ [Fig. 2D].

PB-CSF3R correlated with total leukocyte count $(\rho=0.303, p=0.022)$ and absolute neutrophil count $(\rho=0.431, p=0.01)$. Baseline PB-CSF3R showed moderate sensitivity and specificity for the occurrence of infection and severe neutropenia $(<2000 / \mathrm{cmm}$ and neutrophils $<1000 / \mathrm{cmm})$ with an area under the curve (AUROC) of 0.677 [Fig. 2E] and 0.707 [Fig. 2F], with cut-off values of $\leq 55 \mathrm{pg} / \mathrm{ml}$ and $\leq 58 \mathrm{pg} / \mathrm{ml}$, respectively.

Overall, the results show the lower levels of circulating CSF3R in BM and PB plasma with cirrhosis advancement and association with occurrence infection and leukopenia.

\section{Low CSF3R levels relates to both decreased stem cell population}

\section{and downregulation of surface receptors}

We have earlier reported that the level of HSCs declines in advanced cirrhosis patients. [7] The lower levels of CSF3R receptors could be due to decreased BM progenitor cells. Hence, we enumerated these cells in various classes of cirrhosis patients. As expected, we found lower CD34+ cells in CTP class B and $\mathrm{C}$ than CTP A and controls [Fig. 3A, B]. Further cell assessment revealed that the \% population of CSF3R+ cells out of CD34+ cells also showed a declining trend with advancing cirrhosis [Fig. 3A, C].

The reduction of a stable pool of CD34+ cells could be due to higher G-CSF levels in the circulation. The mobilized HSCs lead to the formation of CFU and BFU in BM. We found that the CFU-GM and BFU-E were more pronounced in CTP A or B patients. [Fig.3 D]. 
This data indicates that the low CSF3R in advanced cirrhosis was related to decreased CD34 cells in BM and downregulation of CSF3R in the remaining CD34 cells in the BM.

\section{CSF3R inhibitory protein: Carcinoembryonic antigen cell adhesion molecule-1 (CEACAM1) in Cirrhosis}

CEACAM1 (biliary glycoprotein) acts as a coinhibitory molecule for CSF3R and downregulates the CSF3RSTAT3 pathway. [16] BM transcriptomics data revealed that the CEACAM1 was upregulated in CTP $C$ than CTP A (2.26; q value $=0.00033)$. On validation, by qRT-PCR, the CEACAM-1 was upregulated in cirrhosis BM compared to controls. [Fig. 4A] On IHC, CEACAM1 positive BM precursor cells were more pronounced in CTP $C$ and $B$ than CTP A and control [Fig. 4B]. Further, the estimation of the circulating level of CEACAM1 in BM plasma [Fig. 4C] and peripheral blood plasma [Fig. 4D] also showed a similar trend $(p<0.001$ in both), with the highest level being in the CTP $C$ class.

The CEACAM1 in BM and peripheral blood plasma levels negatively correlated with the CSF3R $(\rho=-0.668$, $p<0.001$ and $\rho=-0.729, p<0.001$, respectively). Whereas, BM-GCSF and PB-GCSF levels correlated positively with BM-CEACAM1 $(\rho=0.726, p<0.001)$ and PB-CEACAM- $1(\rho=0.719, p<0.001)$, respectively.

CEACAM-1 is expressed mainly in the biliary and colonic epithelium. So we did IHC in this cohort's available liver biopsies $\{n=14$, (Control/CTP A/B/C=6/3/3/2)\}. Interestingly we noted that in cirrhosis, especially in advanced cirrhosis, increased hepatic expression of CEACAM-1. [Fig. S5]

The results suggest the increased levels of CSF3R inhibitory protein CEACAM-1 in advanced cirrhosis.

CEACAM-1 downregulates CSF3R in the Bone marrow precursor cells:

In vitro, bone marrow mononuclear cultured cells treated with CEACAM-1 for 24 hours revealed a significant mean reduction $(-21.8 \%, p=0.031)$ of CD34+CSF3R+ cells compared to untreated cells. [Fig.

4E]

CSF3R downregulation due to CEACAM in BM precursor cells occurs possibly due to lysosomal degradation

Due to elevated CEACAM1 levels, it is likely that the CSF3R receptor may get degraded in lysosomes and cannot be recycled. To test this hypothesis, we assessed the lysosomal contents in sorted BM CD34 cells by CXCR4 immunocytochemistry. We noted that the lysosome contents increased in cirrhosis as compared to controls. Moreover, the incremental changes noted with the advancement of cirrhosis [Fig. 5 A, B]. Further, on flow cytometry, the lysosomes were assessed by TLR 3 in the CD34 cells. CD34+ CSF3RTLR3+ cells showed increasing $(p<0.001)$ [Fig. 5C] and CD34+CSF3R+TLR3- had a declining trend $(p=0.001)$ [Fig 5D] with the severity of liver cirrhosis. Likewise, BM precursor cells show incremental ubiquitin expression with cirrhosis advancement ( $p=0.007)$ [Fig 5E, F].

CD34+ CSF3R- TLR3+ positive cells and ubiquitin positive cells correlated with BM cellular CEACAM-1 $(\rho=0.642, p<0.001$ and $\rho=0.459, p<0.001)$, respectively. 
Thus the increasing lysosomal contents in CD34+ cells and increased ubiquitin in BM indirectly suggest that the CSF3R is degraded, possibly due to elevated CEACAM1.

\section{Phase-2: Validation Studies of CSF3R}

\section{CSF3R and Response to G-CSF in Cirrhosis}

We took the stored plasma of patients $(n=22)$ who received G-CSF treatment in an earlier study. ${ }^{(11)}$ Fourteen were responsive, and eight were nonresponsive to G-CSF Table S4]. The baseline CSF3R levels were lower in BM and PB in patients who eventually did not respond to the therapy [Fig. 6A]. Contrarily, GCSF at baseline was higher in non-responders in comparison to those who responded. [Fig. 6B] Moreover, post GCSF leukocyte count $(\rho=0.48, p=0.02)$, neutrophil count $(\rho=0.42, p=0.043)$ and CD34 cells $/ 20 x$ in liver biopsy $(\rho=0.25, p=0.055)$ correlated with baseline CSF3R in these patients. ROC analysis of CSF3R in peripheral blood identified a cut-off value of $<6.2 \mathrm{pg} / \mathrm{ml}$ for non-response with area under the curve 0.893, $p=0.001$ with a sensivity of $87.5 \%$ and specificity of $85.7 \%$.

\section{CSF3R and Response to G-CSF in Cirrhosis related Neutropenia}

G-CSF therapy was given to 15 patients with neutropenia from June 2019- June 2020. Six of these did not show improvement in leukocyte count. In comparison, it was noted that non-responders had lower CSF3R ( $p=0.015)$ in blood, and of them in four patients, baseline CSF3R was almost undetectable [Fig. 6C] The circulatory G-CSF was higher in non-responders, although it was not statistically significant $(p=0.071)$. [Fig. 6D]

\section{Phase-3: Single nucleotide polymorphism (SNPs) in CSF3R gene}

Variation in the GCSF response could also be due to the genetic polymorphism of CSF3R. So we tested for SNPs of the CSF3R gene. In our study set, no SNPs were detected.

\section{Discussion}

The present study revealed that the G-CSF receptor (CSF3R) decline in liver cirrhosis. CSF3R becomes significantly downregulated in advanced cirrhosis. This reduction of CSF3R was related to hematopoietic niche alterations, lower HSCs count in BM, and reduced expression of CSF3R in remaining HSCs. It could be related to elevated CEACAM-1 in circulation as well as in the BM. On validation, it was noted that those patients who did not respond to G-CSF therapy had decreased CSF3R at baseline.

It is the first human study exploring the lowered CSF3R in liver cirrhosis to the best of our knowledge. We argue that the lowered CSF3R in cirrhosis could cause a non-response or variable response to G-CSF therapy.

G-CSF has been a great success in the setting of chemotherapy-induced leukopenia and as a CD34+ mobilizing agent in BM transplant. Nevertheless, there have been concerns related to GCSF induced 
mobilization. These concerns are broadly related to HSCs niche disruption [17], underlying chronic ailment, i.e., diabetes [18], and genetic polymorphisms of the hematopoietic niche molecules (CSF3R, VCAM). $[19,20]$ HSC niche disruption and underlying chronic ailment issues are interrelated. In advanced cirrhosis, we have shown that HSCs and their niche components are adversely affected. $[7,8]$ Impaired glucose tolerance in diabetes can cause an inadequate response to GCSF. Likewise, another study from our group revealed that BM-MSCs have insulin resistance and impaired bioenergetics in cirrhotic patients. [21] The genetic polymorphisms of CSF3R were not identified in this study. It indicates a limited role of CSF3R polymorphisms and G-CSF therapy-related responses in cirrhosis patients. Thus, in advanced cirrhosis patients, primarily the disruption of stem cell niche and possibly an impaired glucose tolerance at the cellular level could induce a suboptimal response to GSCF.

The downregulation of CSF3R inversely correlated with CEACAM-1 in BM and circulation. CEACAM-1 is a coinhibitory molecule of CSF3R [16]. CEACAM-1 is expressed mainly in the colonic and biliary epithelium, monocytes, NK cells [22], and significantly less in BM. [23] Ductular reaction and metaplastic hepatocytes cirrhosis can cause the excess CEACAM- 1 in circulation. It works through the contact at the cell surface and can act both as a receptor/ligand. [22] CEACAM1 controls immunity and immune activation. [22, 24, 25] Exogenous cues CEACAM-1 in BM might control the CSF3R by a similar mechanism. The contact control explains that it was very prominent in immunohistochemistry and ELISA in CTP-B and C in BM. However, it was mildly upregulated at the BM transcription level. So, CEACAM-1 is not produced by marrow, somewhat peripherally from the diseased liver, and maybe from affected colonic epithelium in cirrhosis. It acts through contact on the cell surface. CSF3R receptor is processed in the endoplasmic reticulum and recycled back to the cell membrane. Alternatively, it is degraded in the lysosomes by ubiquitination. $[3,26]$ Increased expression of TLR3 and ubiquitin and their inverse correlations with CSF3R; indirectly supports a possible lysosomal degradation of CSF3R.

Elevated GCSF could be harmful, especially if it is rapid and 20-50 times of controls, like in severe trauma. $[27,28]$ Cirrhotic patients had elevated G-CSF, and it was more in advanced cirrhosis. Nevertheless, the rise was mild to moderate (2-4 times) in various stages, so it may not be that harmful in cirrhosis. Still persistently raised GSCF induces HSCs to refractory colony formation stages, and the BM pool of HSCs starts declining.

The study has some limitations. First, we did transcriptomics in a limited number of samples. However, we validated our findings at multiple levels to support the granular change in CSF3R levels in liver cirrhosis patients. Secondly, a potential selection bias could be because we analyzed those cirrhotic patients who underwent bone marrow examination. However, as described in the methods section, the clinical indications across the CTP classes were similar, and we consecutively enrolled all cirrhotic patients with BM examinations. Hence, the bias was minimized.

Nevertheless, this study has important clinical significance. It shed light on a few mechanistic aspects, which have significant therapeutic implications. CSF3R assessment could be useful for those patients who could get benefit from G-CSF therapy. Thus, it will help the clinicians to make appropriate decisions 
for GCSF treatment. CS3R improvement can be achieved by humanized anti-CEACAM-1 IgG4 antibodylike MK-6018 $[24,29]$ in the remaining HSCs of the BM of cirrhotic patients.

\section{Declarations}

\section{Compliance with ethical standards}

\section{Conflict of Interest: None}

Ethical approval: All procedures performed in studies involving human participants were in accordance with the ethical standards of the insti-tutional and/or national research committee and with the 1964 Helsinki declaration and its later amendments or comparable ethical standards (and/or in case humans were involved).

\section{References}

1. Paczkowska E, Kucia M, Koziarska D, et al. Clinical evidence that microscopic embryonic-like stem cells are mobilized into peripheral blood in patients after stroke. Stroke. 2009; 40(4):1237-1244.

2. Gehling UM, Willems $M$, Schlagner $K$, et al. mobilization of hematopoietic progenitor cells in patients with liver cirrhosis. World J Gastroenterol. 2010; 16(2):217-224.

3. Dwivedi P, Greis KD. Granulocyte colony-stimulating factor receptor signaling in severe congenital neutropenia, chronic neutrophilic leukemia, and related malignancies. Exp Hematol. 2017; 46:9-20.

4. Kedarisetty CK, Anand L, Bhardwaj A, 'et al. Combination of Granulocyte colony-stimulating factor and erythropoietin improves outcomes of patients with decompensated cirrhosis. Gastroenterology. 2015; 148(7):1362-70.

5. Garg V, Garg H, Khan A, Trehanpati N, Kumar A, Sharma BC, et al. Granulocyte colony-stimulating factor mobilizes CD34(+) cells and improves survival of patients with acute-on-chronic liver failure. Gastroenterology. 2012; 142(3):505-512.

6. Newsome PN, Fox R, King AL, et al. Granulocyte colony-stimulating factor and autologous CD133positive stem-cell therapy in liver cirrhosis (REALISTIC): an open-label, randomised, controlled phase 2 trial. Lancet Gastroenterol Hepatol. 2018; 3(1):25-36.

7. Bihari $C$, Anand $L$, Rooge $S$, et al. Bone marrow stem cells and their niche components are adversely affected in advanced cirrhosis of the liver. Hepatology. 2016; 64(4):1273-1288.

8. Bihari C, Lal D, Thakur M, et al. Suboptimal Level of Bone-Forming Cells in Advanced Cirrhosis are Associated with Hepatic Osteodystrophy. Hepatol Commun. 2018; 2(9):1095-1110.

9. Lee SG. A complete treatment of adult living donor liver transplantation: a review of surgical technique and current challenges to expand indication of patients. Am J Transplant. 2015; 15(1):1738. 
10. Hisada $M$, Zhang $X$, Ota $Y$, et al. Fibrosis in small syngeneic rat liver grafts because of damaged bone marrow stem cells from chronic alcohol consumption. Liver Transpl. 2017; 23(12):1564-1576.

11. Anand L, Bihari C, Kedarisetty CK, et al. Early cirrhosis and a preserved bone marrow niche favour regenerative response to growth factors in decompensated cirrhosis. Liver Int. 2019; 39(1):115-126.

12. Lv Y, Yee Lau W, Wu H, et al. causes of peripheral cytopenia in hepatitic cirrhosis and portal hypertensive splenomegaly. Exp Biol Med (Maywood). 2017; 242(7):744-749.

13. Varadarajan A, Lal D, Kapil R, Bihari C. Bone marrow dyspoiesis associated with severe refractory anaemia in liver cirrhosis Frontline Gastroenterology 2020. doi: 10.1136/flgastro-2019-101350.

14. Choi PC, Kim HJ, Choi WH, et al. Model for end-stage liver disease, model for end-stage liver diseasesodium and Child-Turcotte-Pugh scores over time for the prediction of complications of liver cirrhosis. Liver Int. 2009;29(2):221-6.

15. Pareja E, Cortes M, Hervás D, et al. A score model for the continuous grading of early allograft dysfunction severity. Liver Transpl. 2015; 21(1):38-46.

16. Hosomi S, Chen Z, Baker K, et al. CEACAM1 on activated NK cells inhibits NKG2D-mediated cytolytic function and signaling. Eur J Immunol. 2013; 43(9):2473-2483.

17. Lucas $D$, Scheiermann $C$, Chow A, et al. Chemotherapy-induced bone marrow nerve injury impairs hematopoietic regeneration. Nat Med 2013; 19:695-703.

18. Fadini GP, Albiero M, Vigili de Kreutzenberg S, et al. diabetes impairs stem cell and proangiogenic cell mobilization in humans. Diabetes Care 2013; 36:943-9.

19. Bendall LJ, Bradstock KF. G-CSF: From granulopoietic stimulant to bone marrow stem cell mobilizing agent Cytokine \& Growth Factor Reviews 2014; 25:355-367.

20. Camurdanoglu BZ, Esendagli G, Ozdemir E, Canpinar H, Guc D, Kansu The effect of granulocyte colony stimulating factor receptor gene missense single nucleotide polymorphisms on peripheral blood stem cell enrichment. Cytokine 2013; 61(2): 572-577.

21. Kumar D, Maheshwari D, Shubham S, et al. Bone marrow-mesenchymal stem cells of cirrhosis patients show insulin resistance and bioenergetic exhaustion. Journal of Hepatology 2019; 70(1):e807-e808.

22. Gray-Owen SD, Blumberg RS. CEACAM1: contact-dependent control of immunity. Nat Rev Immunol $2006 ; 6: 433-446$.

23. https://www.proteinatlas.org/ENSG00000079385-CEACAM1/tissue.

24. Suda T, Tatsumi T, Nishio A, et al. CEACAM-1 Is Associated With the Suppression of Natural Killer Cell Function in Patients With Chronic Hepatitis C. Hepatol Commun. 2018; 2(10):1247-1258.

25. Khairnar V, Duhan V, Patil AM, et al. CEACAM1 promotes $C D 8^{+} \mathrm{T}$ cell responses and improves control of a chronic viral infection. Nat Commun 2018; 9: 2561.

26. Lochab S, Pal P, Kapoor I, et al. E3 ubiquitin ligase Fbw7 negatively regulates granulocytic differentiation by targeting G-CSFR for degradation, Biochimica et Biophysica Acta (BBA) - Molecular Cell Research. 2013; 1833 (12):2639-2652. 
27. Cook KM, Sifri ZC, Baranski GM, et al. The role of plasma granulocyte colony stimulating factor and bone marrow dysfunction after severe trauma. J Am Coll Surg. 2013; 216(1):57-64.

28. Laurenti E, Göttgens B. From haematopoietic stem cells to complex differentiation landscapes. Nature. 2018; 553(7689):418-426.

29. Markel G, Sapir Y, Mandel I, et al. Inhibition of the novel immune checkpoint CEACAM1 to enhance anti-tumor immunological activity. Journal of Clinical Oncology 2016; 34: 3044-3044.

\section{Figures}



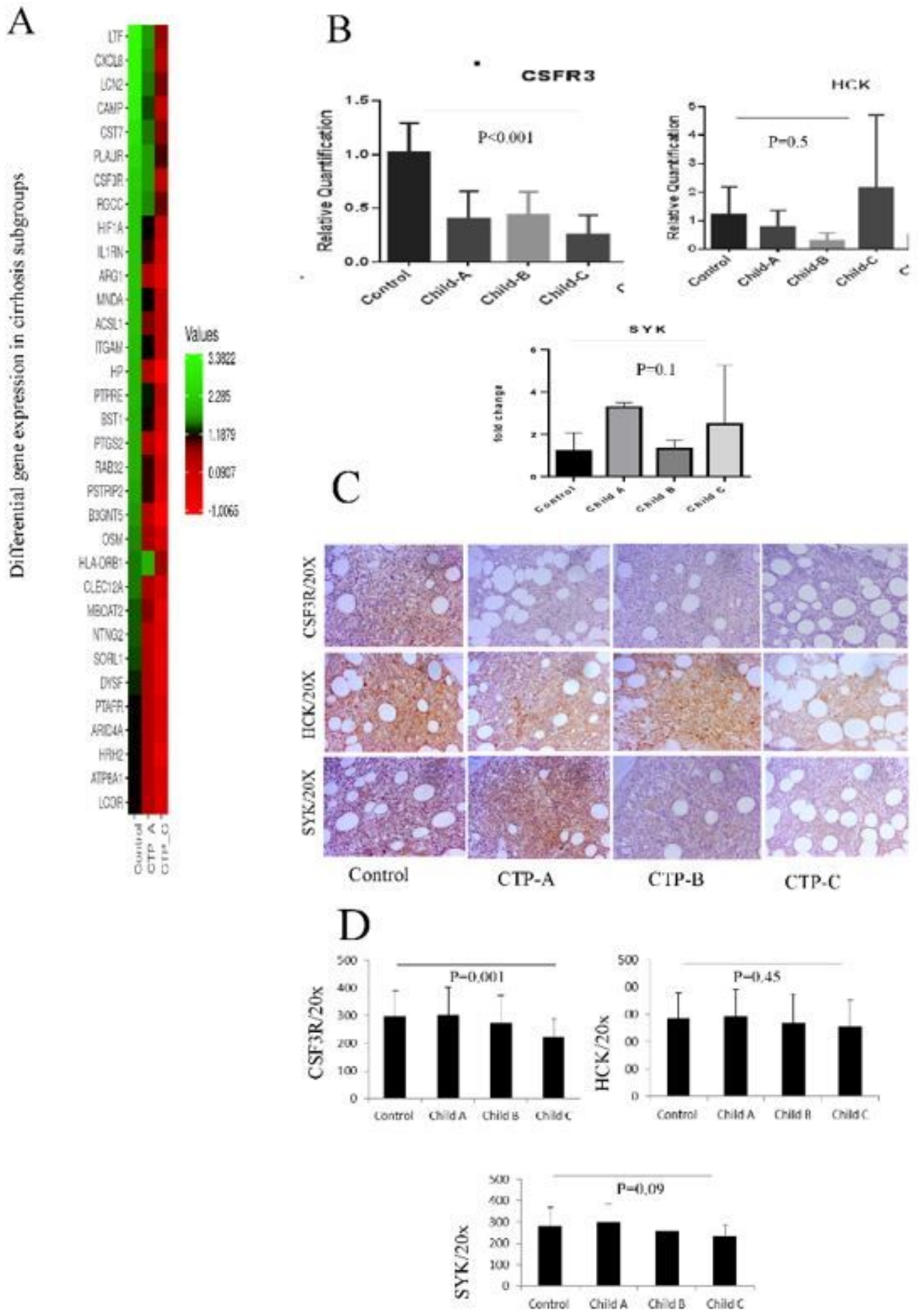

Figure 1

CSF3R in the bone marrow: A. Heatmap showing uniformly downregulated gene across the groups in bone marrow biopsies- comparing control-Child-Turcott-Pugh (CTP) class $A$ and $C, n=5$ each. $B$. Quantitative RT-PCR of mRNA of colony-stimulating factor receptor 3 (CSF3R), hematopoietic cell kinase (HCK), and splenic kinase (Syk), in the bone marrow biopsies (mean $\pm S D), n=5$ each. C. Representative images of Immunohistochemistry (IHC) expression of CSF3R, HCK, SYK in the bone marrow biopsies of 
control and CTP-A, B, C classes of cirrhosis, all cases. D. Graphical representation of the average number of CSF3R, HCK, and Syk positive cells/20x in control and CTP-A, B, C classes of cirrhosis presented as mean $\pm S D$, all cases.
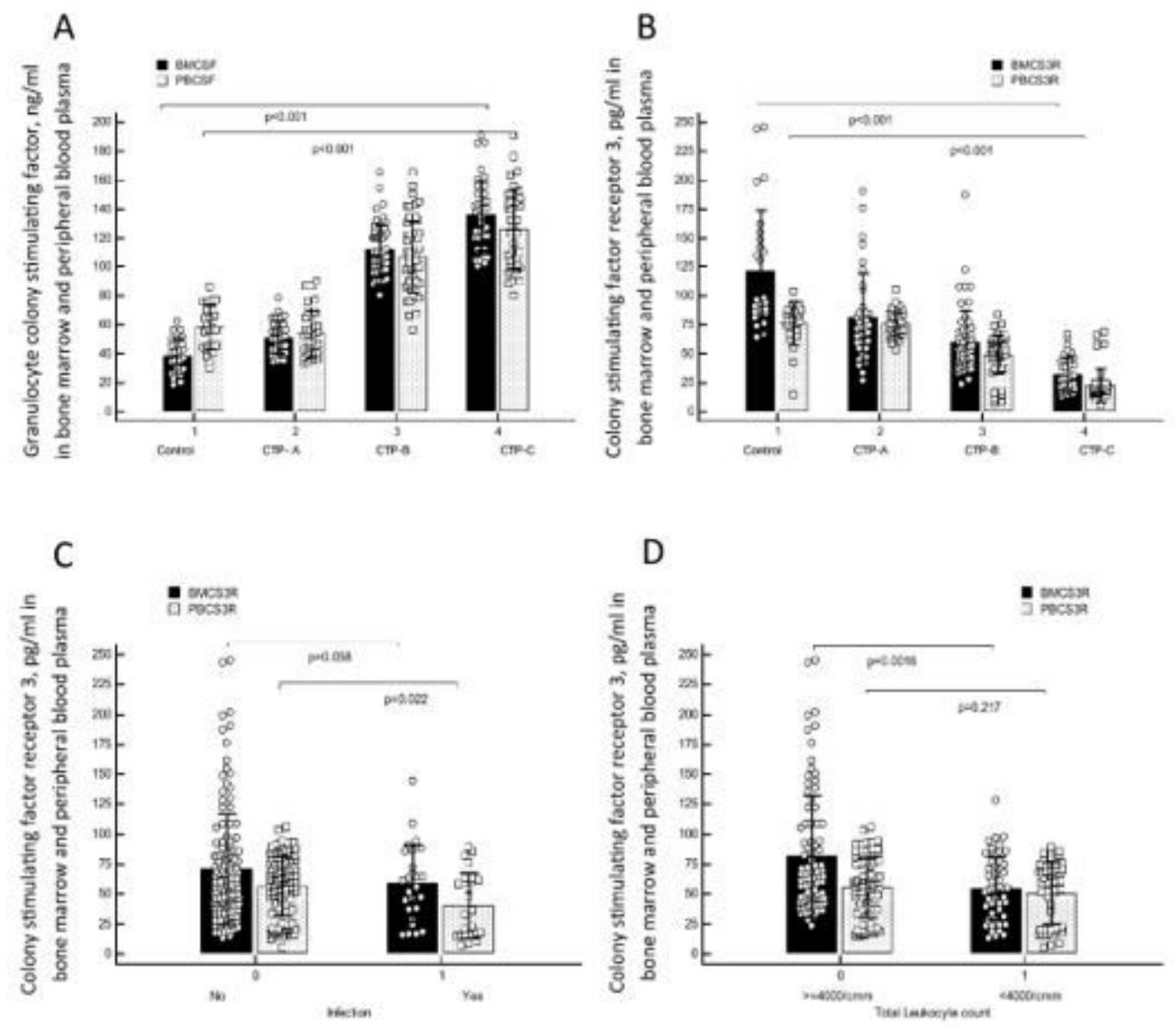

$E$

$\mathrm{F}$
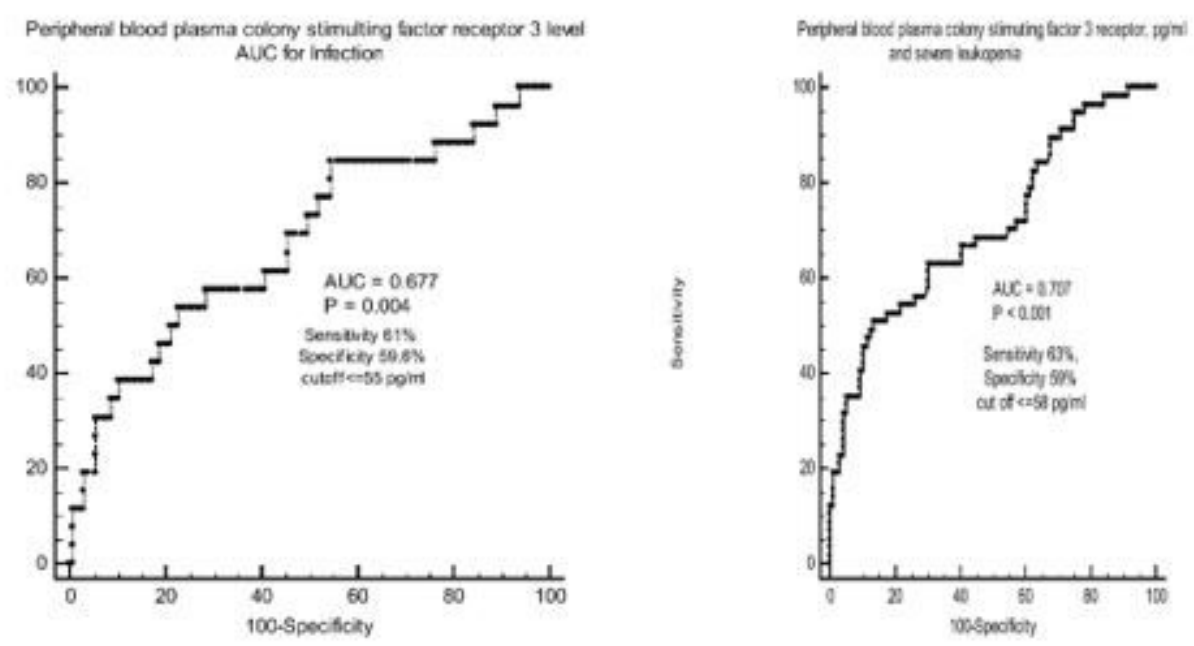

Figure 2

GSCF and CSF3R in the circulation: A. Granulocyte colony-stimulating factor (G-CSF) levels in the bone marrow plasma and peripheral blood plasma of control and cirrhosis cases according to CTP A, B, C 
(mean $\pm S D)$. B. Colony-stimulating factor receptor (CSFR) levels in the bone marrow plasma and peripheral blood plasma of control and cirrhosis cases according to CTP A, B, C (mean $\pm S D)$. C. Comparison of baseline colony-stimulating factor receptor (CSFR) levels in the bone marrow plasma and peripheral blood plasma of those cirrhotic patients who had naturally acquired infection during follow-up, and D. of those cirrhotic patients who had leukopenia and with normal total leukocyte count. E. The area under the curve CSF3R level in peripheral blood for infection and F. for severe leukopenia. [Included all cases]

A
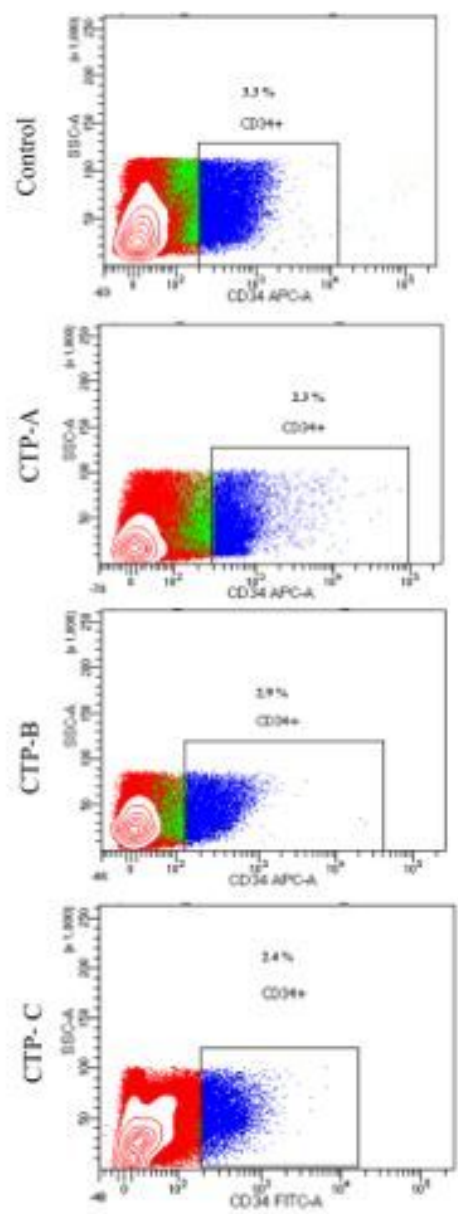

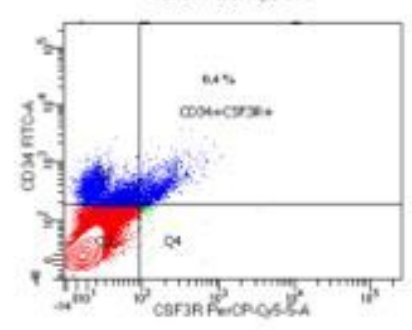

CD34 CSF3R
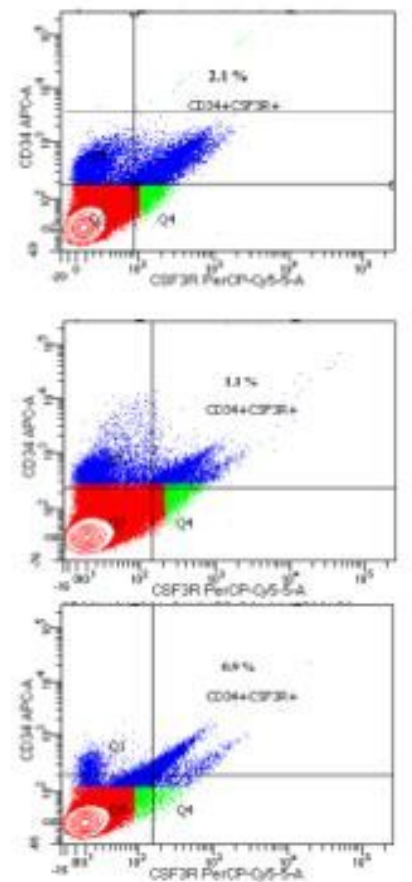

B

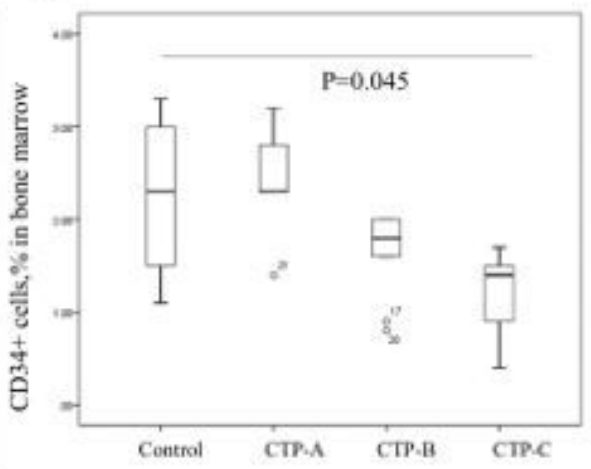

$\mathrm{C}$

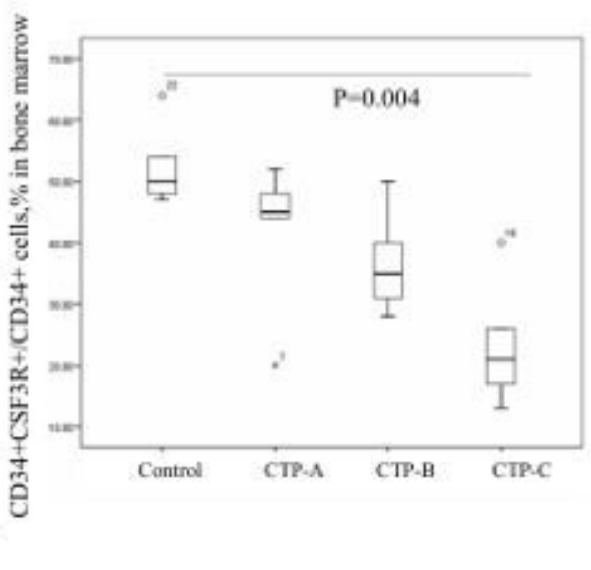

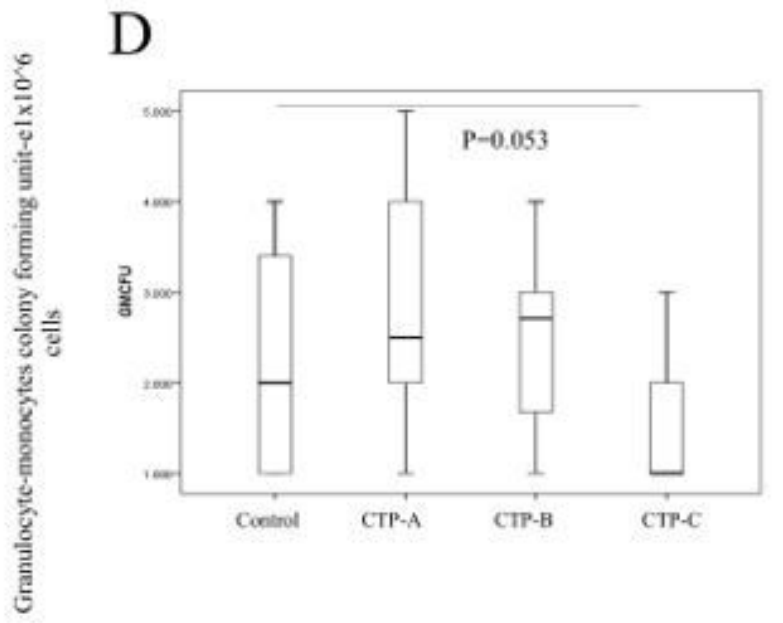

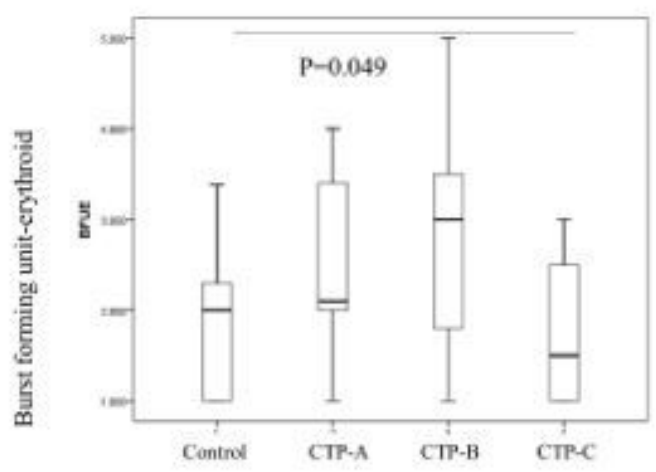


Figure 3

Status of CSF3R in the BM precursor cells: A. Representative images of flow cytometry of bone marrow aspirate samples with acquired CD34+ cells (\%) and CSF3R+CD34+ cells (\%) in control and cirrhosis (CTP classes $A, B, C), n=25$ each. B. Distribution of CD34+ cells \% in control and cirrhosis (CTP classes $A, B, C$ ) \{median (interquartile range)\}, $\mathrm{n}=25$ each. $\mathrm{C}$. Distribution of $\mathrm{CSF} 3 \mathrm{R}+\mathrm{CD} 34+(\%)$ out of total bone marrow C34+ cells \{median (interquartile range), $\mathrm{n}=25$ each. D. Distribution of GM-CFU and BFU-E across the study groups $\{$ median (interquartile range) $\}, \mathrm{n}=10$ each.

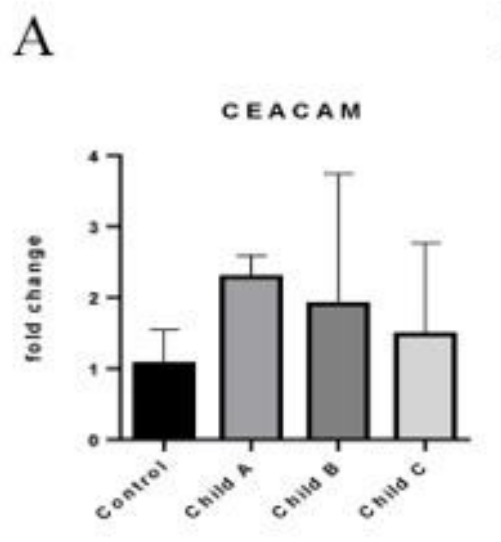

$\mathrm{C}$
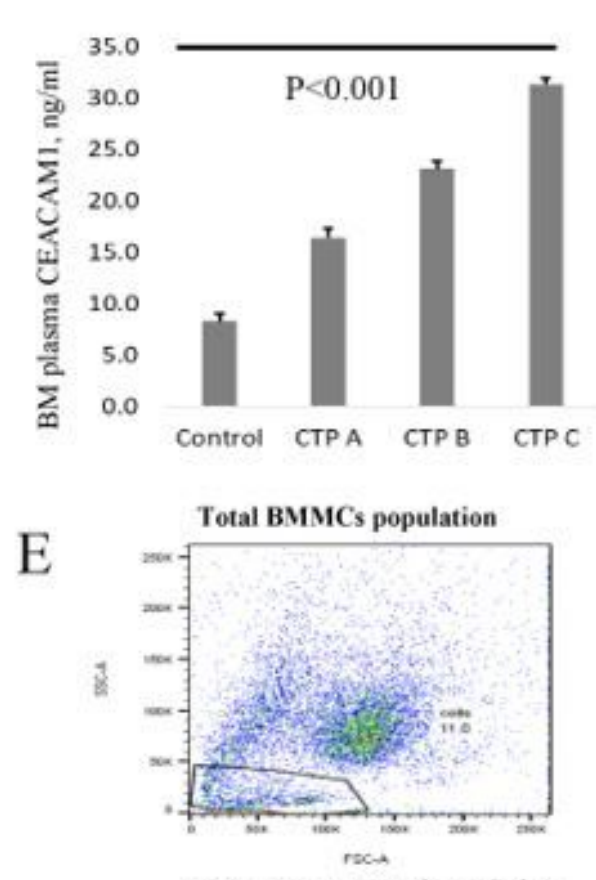

CEACAM 1 untreated population

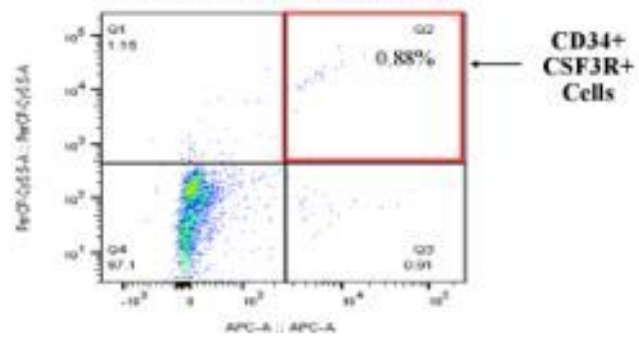

D
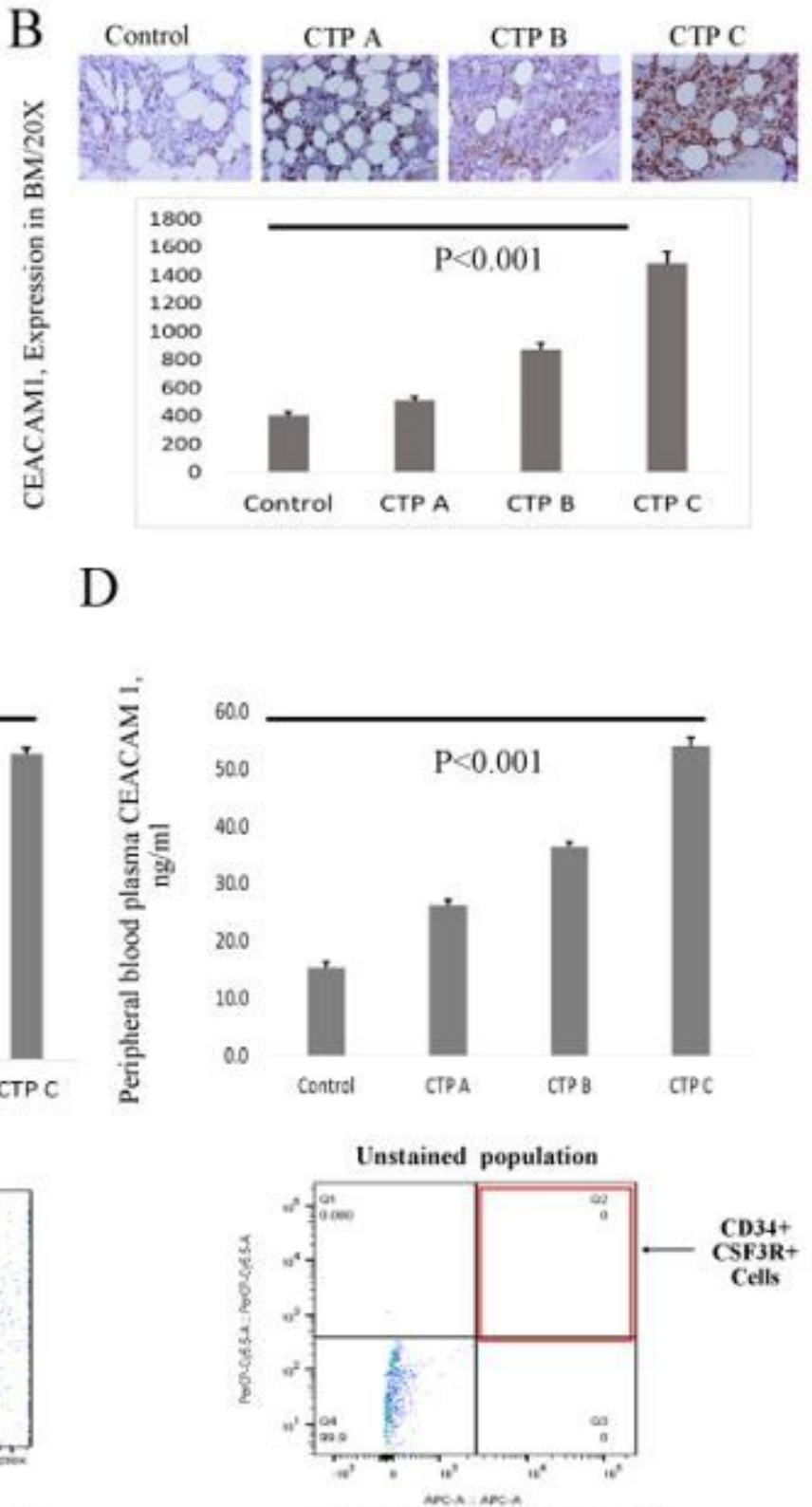

CEACAM 1 treated population

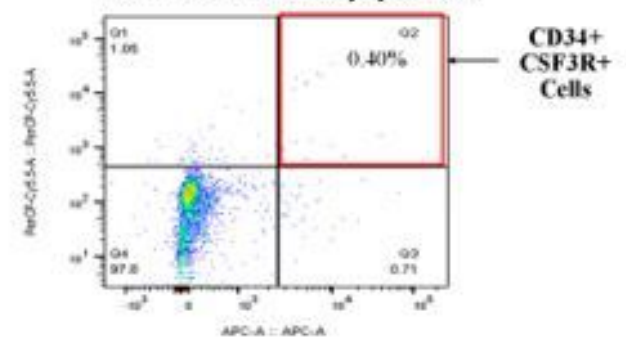

Page 16/19 


\section{Figure 4}

A: CEACAM-1 in cirrhosis and its association with CSF3R: Expression level m RNA of CEACAM-1 gene in the bone marrow biopsies of control cirrhosis classes (mean $\pm S D$ ), $n=5$. B. Representative Images of CEACAM-1 positive bone marrow cells (arrow) in the biopsies of control and cirrhosis classes (20x). C. Graphical representation of distribution CEACAM-1+ cells in bone marrow across the study groups $(m e a n \pm S D)$. D. CEACAM-1 levels in the bone marrow plasma of control and cirrhosis classes (mean $\pm S D)$, and $\mathrm{E}$. representative images of flow cytometry of bone marrow mononuclear cultured cells are displaying the difference in CD34+CSF3R+ cells without CEACAM-1 and with CEACAM-1 treatment for 24 hours, $n=5$. 
A

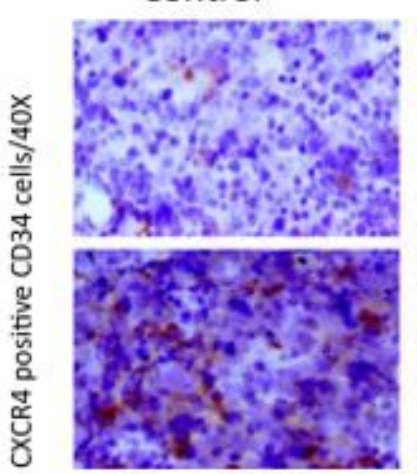

CTP B

\section{C}

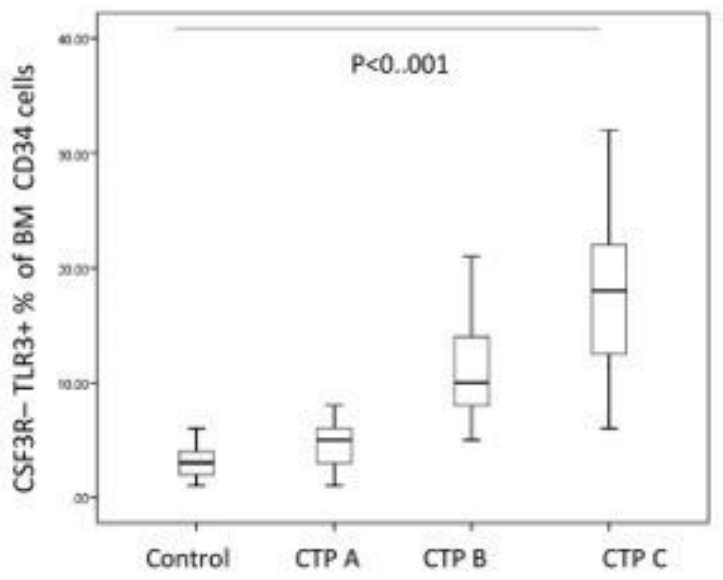

E

Control

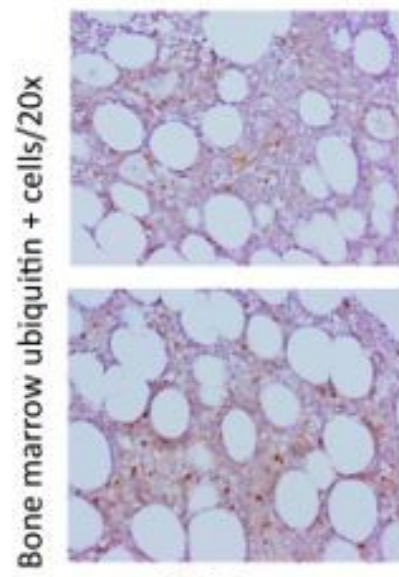

CTP B
CTP A

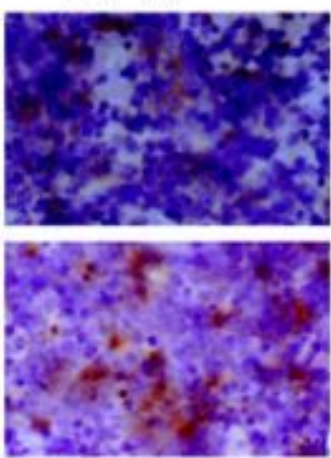

CTP C
CTP A

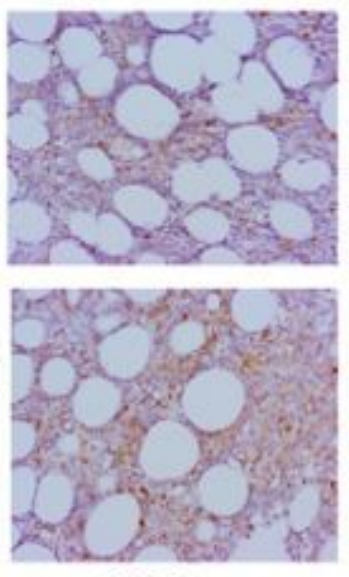

B

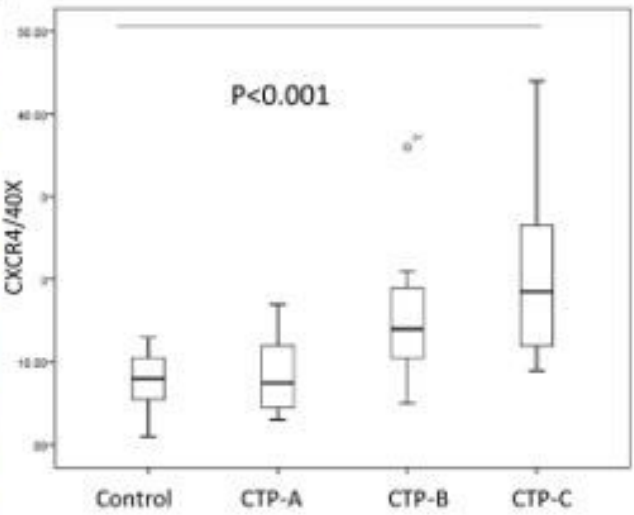

D

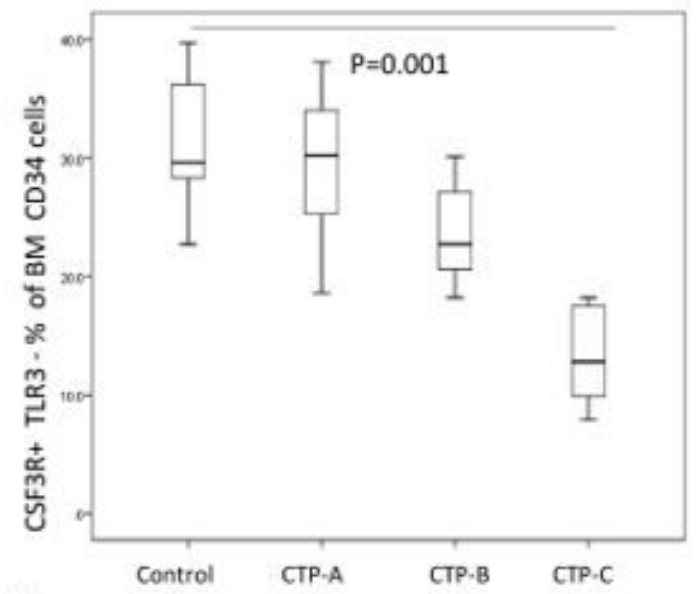

F

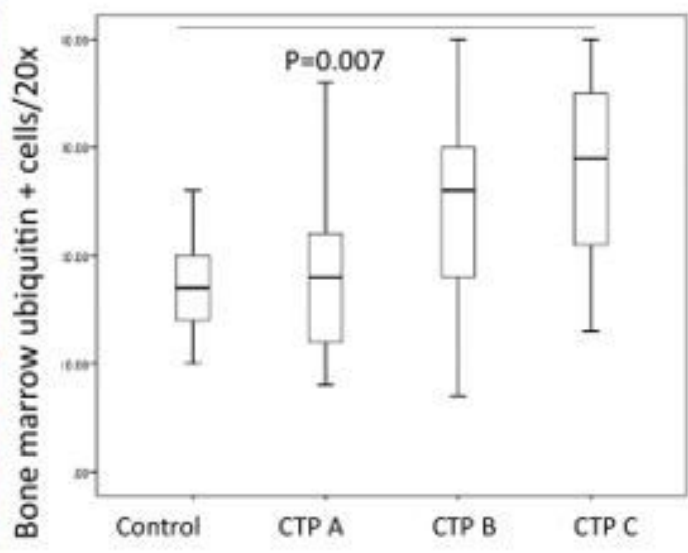

\section{Figure 5}

Increased Lysosomes in the bone marrow cells of Cirrhosis patients: A. CXCR4 positive cells in the cytospin smear of sorted CD34 cells of bone marrow in the control and cirrhosis classes (Giemsa, 20x), and $B$. its graphical distribution, \{median (interquartile range)\}, $\mathrm{n}=10$ each. C. Graphical distribution of CSF3R-TLR3+ cells out of CD34 cells, and D. CSF3R+TLR3- of CD34 cells in control and cirrhotic classes, \{median (interquartile range)\}, $\mathrm{n}=25$ each. E. Representative images of ubiquitin-positive cells/20x in the 
bone marrow of study groups (20x) and F. graphical representation of these cells across the study groups, \{median (interquartile range)\}, all cases.

A

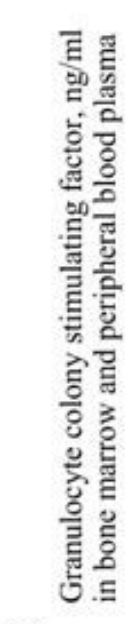

$\mathrm{C}$
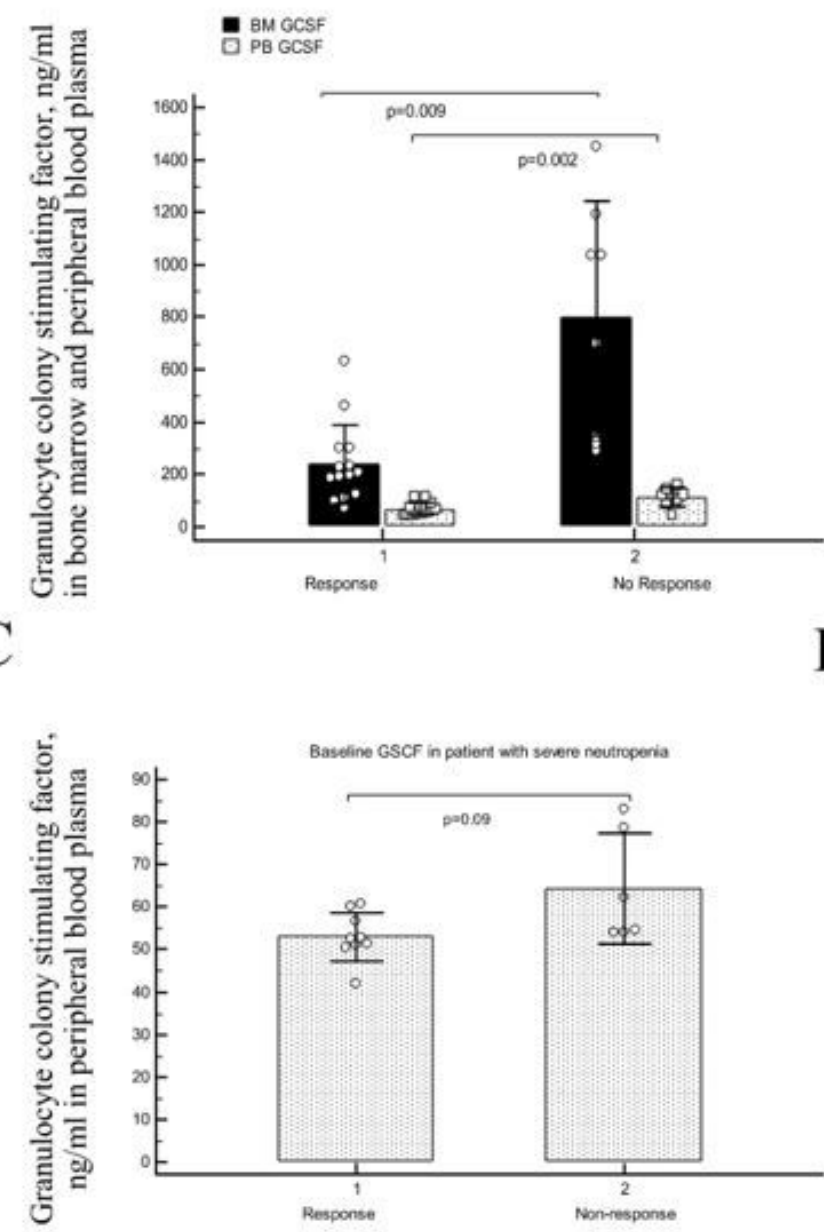

B
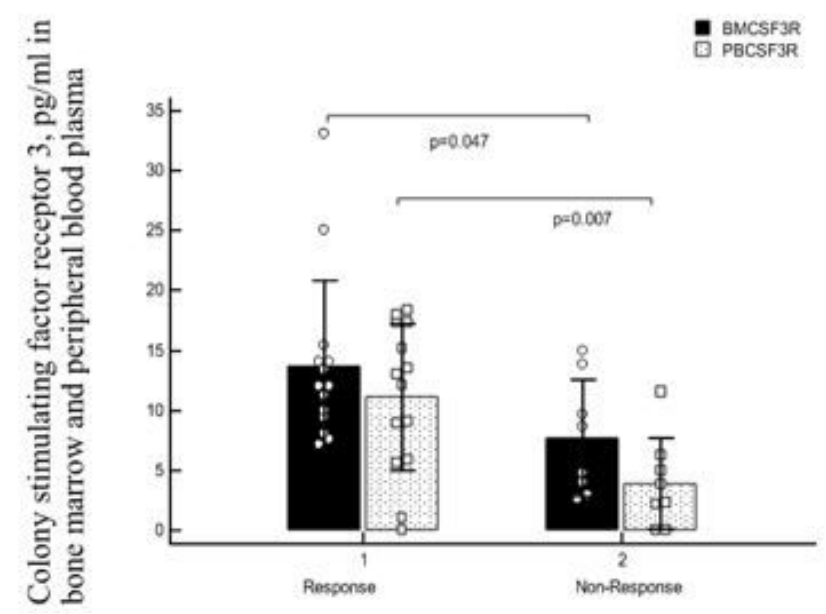

$\mathrm{D}$

口 PB CSF3R
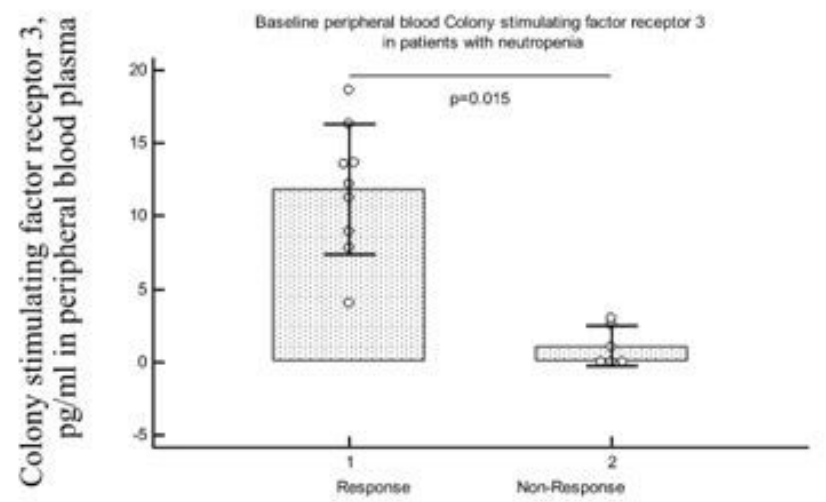

\section{Figure 6}

Relation of Baseline CSF3R with a response to GSCF and Post-transplant leukopenia and infections: Comparison of A. granulocyte colony-stimulating factor B. CSF3R levels in the bone marrow and peripheral blood plasma cirrhosis patients who responded to GSCF as regenerative therapy vs. nonresponders (mean $\pm S D$ ). Comparison of baseline C: GCSF and D. CSF3R in the peripheral blood plasma of cirrhosis patients who responded to GSCF treatment for neutropenia vs. non-responders (mean $\pm S D$ ). 